\title{
Altered expression levels of HLA class II and costimulatory molecules on circulating monocytes from patients with cervical intraepithelial neoplasia and squamous cervical cancer
}

\author{
TING FENG $^{1 *}$, JIAN-HONG ZHOU ${ }^{1 *}$, JIA LIU ${ }^{1}$, FENG YE $^{2}$, WEIGUO LU ${ }^{1}$ and XING XIE ${ }^{1}$ \\ ${ }^{1}$ Department of Gynecologic Oncology, and ${ }^{2}$ Central Laboratory, Women's Hospital, \\ School of Medicine, Zhejiang University, Hangzhou 310006, P.R. China
}

Received February 18, 2012; Accepted June 15, 2012

DOI: $10.3892 / \mathrm{mmr} .2012 .1068$

\begin{abstract}
The aim of this study was to investigate the role of the cell surface expression levels of HLA class I and II molecules, the costimulatory molecules CD80/B7-1 and CD86/B7-2, and the adhesion molecules CD54 and CD58 during cervical carcinogenesis. The expression levels of MHC class I and II molecules, the costimulatory molecules CD80/ B7-1 and CD86/B7-2 and the adhesion molecules CD54 and CD58 on $\mathrm{CD}_{14}{ }^{+}$peripheral blood monocytes (PBMs) from 21 cases of cervical intraepithelial neoplasia (CIN) II-III, 51 squamous cervical carcinomas (SCCs) and 18 healthy controls were analyzed using flow cytometry analysis. We found increased expression levels of HLA-DR $(\mathrm{p}=0.000)$, HLA-DQ $(p=0.000), C D 86 / B 7-2(p=0.002)$ and CD58 $(p=0.000)$ on PBMs from patients with SCC and CIN II-III, compared with healthy control subjects, whereas no significant difference existed in the expression levels of HLA class I antigens, HLA-DP CD80/B7-1 and CD54. Upregulated expression levels of HLA-DR, HLA-DQ, CD86/B7-2 and CD58 were associated with disease progression, indicating that an increased expression of HLA-DR, HLA-DQ, CD86/B7-2 and CD58 on PBMs may be correlated with the evolution of cervical cancer.
\end{abstract}

\section{Introduction}

Detection and treatment of precursor lesions have provided the basis for cervical screening programs, which have successfully reduced the incidence of and mortality from cervical cancer in the USA and European countries. However, $80 \%$ of cervical cancer mortalities worldwide occur in countries where there

Correspondence to: Dr Xing Xie, Department of Gynecologic Oncology, Women's Hospital, School of Medicine, Zhejiang University, 2 Xueshi Rd, Hangzhou 310006, P.R. China

E-mail: xiex@zju.edu.cn

*Contributed equally

Key words: cervical neoplasm, monocyte, HLA class I, HLA class II, CD80/B7-1, CD86/B7-2, CD54, CD58 are insufficient health care resources to treat invasive disease or establish cervical cancer screening programs (1). Even with optimal treatment, $40 \%$ of patients treated for invasive cervical cancer are likely to relapse and succumb to the disease. It takes a long time to develop from cervical intraepithelial neoplasia (CIN) to invasive cancer, accompanied by HPV infection (2).

The escape of tumor cells from host immunosurveillance is known as one of the major mechanisms enabling unrestrained neoplastic cell growth and the formation of metastases. This immune escape is thought to be supported either by a mechanism of defense exerted by the tumor cells themselves and/or by an impaired function of the host immune system (3). Our previous study revealed that increased expression of HLA-DR by tumor cells may be related to the evolution of cervical cancer (4). Since professional antigen-presenting cells (APCs) are known to be one of the most important inducers of an antigen-specific immune response, their potentially defective function causes a strong impairment of immunosurveillance in tumor-bearing hosts (5). Professional APCs include dendritic cells (DCs), B cells and macrophages. Two of these cell types may originate from the peripheral blood monocytes (PBMs): Macrophages by extravasal migration in vivo, and DCs in vitro when cultured with additional cytokines (6). However, the dendritic cells are the most effective APCs in the induction of primary immune responses and are considered the best vehicle for the delivery of tumor-associated antigens in the immunotherapy of cancer patients. Macrophages are important in the immune defense against bacterial and viral infections, as well as against tumor cells. Their progenitors, the PBMs, have been shown to exert a so-called monocyte-mediated tumoricidal activity $(7,8)$. This direct cytotoxic effect requires a monocyteto-tumor-cell contact and is independent of MHC class I or II expression or presentation of tumor-associated antigenic peptides. However, little is known about the potency of PBMs to sufficiently induce an antigen-specific immune response. While generally expressing the MHC class II cell surface molecules HLA-DR, HLA-DQ and HLA-DP, PBMs show only a low surface expression of co-stimulatory molecules. Nevertheless, the cell surface expression of HLA-DR has been shown to be downregulated on PBMs from polytraumatic patients developing severe sepsis compared with patients with non-septicemic outcome (9). In the present study, we 
investigated the expression of the HLA class II molecules HLA-DR, HLA-DQ and HLA-DP on PBMs from patients with CIN II-III and squamous cervical cancer compared with healthy controls. Moreover, we analyzed the cell surface expression of HLA class I molecules, the costimulatory molecules CD80/B7-1 and CD86/B7-2, and the adhesion molecules CD54 (ICAM-1) and CD58 (LFA-3).

\section{Materials and methods}

Patients. The study subjects were recruited between November 2004 and April 2006 from the Women's Hospital, School of Medicine, Zhejiang University, China. None of the patients received chemotherapy or radiotherapy. After informed consent was obtained, blood was drawn from the study subjects, which included 21 patients with cervical lesions and moderate dysplasia (CIN II) or severe dysplasia (CIN III) with a mean age of $38.3 \pm 9.6$ years, and 51 invasive squamous cell carcinoma (SCC) patients with a mean age of $44.1 \pm 9.7$ years. Blood samples from 18 healthy controls with a mean age of $43.6 \pm 8.3$ years were kindly provided by the Department of Physical Examination of Zhejiang Traditional Medicine Hospital. The controls had undergone regular physical and laboratory examinations.

Monoclonal antibodies. The anti-pan-HLA class I (TU149) with the fluorescein isothiocyanate (FITC)-conjugated $\mathrm{mAb}$, CD14 (TUK4) with FITC-conjugated mAb, CD80/B7-1 mAb (MEM2-33) with FITC-conjugated mAb, CD86/B7-2 (BU63) with the phycoerythrin (PE)-conjugated $\mathrm{mAb}$ and CD54/ICAM-1 (MEM-111) with PE-conjugated mAb were obtained from Invitrogen (San Diego, CA, USA). MAbs recognizing FITC-conjugated HLA-DQ (TÜ169), PE-conjugated HLA-DR (TÜ36) and FITC-conjugated CD58/LFA-3(1C3) were purchased from PharMingen (San Diego, CA, USA). The anti-HLA-DP (B7/21) with PE-conjugated $\mathrm{mAb}$ was purchased from Universal Biologicals (Cambridge, UK).

Flow cytometry. Peripheral blood mononuclear cells (PBMCs) were isolated from freshly obtained heparinized blood samples by Ficoll-Hypaque density gradient centrifugation. Following $60 \mathrm{~min}$ of incubation at $4^{\circ} \mathrm{C}$ with unspecific rabbit $\operatorname{IgG}(5 \mu \mathrm{g} / \mathrm{ml}$ PBS $)$ in order to block non-specific Fc-receptor binding on monocytes, cells were incubated for $20 \mathrm{~min}$ at $4^{\circ} \mathrm{C}$ with different $\mathrm{mAbs}$ in $\mathrm{PBS} / 0.5 \%$ bovine serum albumin (BSA) $/ 0.02 \%$ sodium azide. The concentrations of mAbs used were determined according to the manufacturer's instructions. Unspecific mouse IgG antibody was used as a control. Stained samples were analyzed using a Coulter Epics XL 2 cytometer with the system II software (Coulter Electronics, Miami, FL, USA). Peripheral blood lymphocytes (PBLs) and PBMs were distinguished by forward and side light scatter properties. $\mathrm{CD} 14^{+}$cells were gated as the cell population of interest. These cells were analyzed for their expression of surface markers by comparing their fluorescence staining with specific mAb versus staining with unspecific isotype-matched $\mathrm{mAb}$. The data were shown as the percentage of the positive cell population.

Statistical analysis. Statistical analysis was performed using SAS 8.0. The Kruskal Wallis test, Bonferroni post hoc test and ANOVA analysis were used for statistical comparisons. Normality of the data was tested using the Kolmogorov-Smirnov test, revealing the age data as normally distributed, whereas the other data were skewed. Differences with a value less than 0.05 were considered statistically significant.

\section{Results}

Patient analysis. $\mathrm{CD}_{14}^{+}$peripheral blood mononuclear cells from 21 patients with moderate CIN II or CIN III and 51 patients with SCC, as well as 18 healthy controls, were analyzed for the cell surface expression of HLA class I and II antigens, CD80/B7-1, CD86/B7-2, CD54 and CD58.

Altered expression of HLA class II antigens. The cell surface expression of the class II molecules HLA-DR $(\mathrm{p}=0.000)$ and HLA-DQ ( $\mathrm{p}=0.000)$ was increased on PBMs from patients with SCC and CIN II-III compared with the healthy control subjects (Table I), whereas there was no significant change in the expression of HLA-DP in patients with SCC and CIN II-III comparied with the controls. However, a markedly increased expression of HLA-DQ $(\mathrm{p}=0.002)$ was observed in patients with CIN II-III compared with SCC, whereas no significant differences were observed between CIN II-III and SCC in regard to the expression of HLA-DR and HLA-DP (Table I).

No significant changes in HLA class I expression and CD54 (ICAM-1). In contrast to HLA class II molecules, the expression of HLA class I on PBMs showed no significant changes in patients with SCC and CIN II-III compared with the healthy control subjects (Table I). The number of PBMs expressing ICAM-1 was found to have no significant differences among the three groups (Table I).

Increased expression of CD86/B7-2 and CD58. The cell surface expression of CD86/B7-2 (p=0.002) and CD58 ( $\mathrm{p}=0.000)$ was increased on PBMs from patients with SCC and CIN II-III compared with healthy control subjects (Table I), but there was no significant change between SCC and CIN II-III patients. The expression of CD80/B7-1 was found to have no significant differences among the three groups (Table I).

\section{Discussion}

A defective function of APCs is known to cause a reduced antigen-specific immune response leading to an impaired recognition and eradication of malignant cells. A decreased antigen-presenting function of DCs has been described in breast cancer patients (10). Despite a discrepancy in frequency, DCs from patients with early and advanced breast cancer exhibit a reduced expression of CD86 and HLA-DR and decreased immunostimulatory abilities. An association was found between the impaired ability of patients' DCs to stimulate allogeneic $\mathrm{T}$ cells associated with the reduced cell surface expression of HLA class II and costimulatory B-7 molecules (11). Although the functional impact of PBMs for the antigen-specific antitumoral immunosurveillance and the defense have yet to be clarified, defects in the antigen-presenting function of PBMs may impair these mechanisms in cancer patients. Previous studies have described the decreased 
Table I. Expression levels of HLA-I, HLA-II, CD80, CD86, CD54 and CD58 in cervical intraepithelial neoplasia and squamous cervical carcinoma on PBMs $[\mathrm{M}(\mathrm{QR})]$.

\begin{tabular}{|c|c|c|c|c|c|c|c|c|c|}
\hline Group & $\mathrm{N}$ & HLA-I & HLA-DR ${ }^{\mathrm{a}}$ & HLA-DP & $\mathrm{HLA}^{-D Q^{\mathrm{b}}}$ & CD80 & $\mathrm{CD} 86^{\mathrm{c}}$ & CD54 & $\mathrm{CD}^{\mathrm{d}}{ }^{\mathrm{d}}$ \\
\hline Controls & 18 & $100(0)$ & $78.3(19.3)$ & $62.3(17.5)$ & $20.9(15.5)$ & $5.4 \quad(3.6)$ & $28.1(11.2)$ & $52.4(26.3)$ & $90.2(9.6)$ \\
\hline CIN & 21 & $100(0)$ & $98.2 \quad(2.6)$ & $87.9(29.8)$ & $69.8(38.3)$ & $7.9 \quad(5.5)$ & $63.1(52.7)$ & $82.1(37.8)$ & $99.3(0.9)$ \\
\hline SCC & 51 & $100(0)$ & $98.1(10.7)$ & $77.4(46.7)$ & $38.7(28.0)$ & $6.2(10.8)$ & $55.8(57.6)$ & $52.4(52.1)$ & $99.5(2.5)$ \\
\hline \multicolumn{10}{|l|}{ P-value } \\
\hline Control vs. CIN & & NS & 0.000 & NS & 0.000 & NS & 0.000 & NS & 0.000 \\
\hline Control vs. SCC & & NS & 0.000 & NS & 0.000 & NS & 0.010 & NS & 0.000 \\
\hline CIN vs. SCC & & NS & NS & NS & 0.002 & NS & NS & NS & NS \\
\hline
\end{tabular}

CIN, cervical intraepithelial neoplasia; SCC, squamous cervical carcinoma; NS, not significant; PBMs, peripheral blood monocytes. ${ }^{\mathrm{a} C o n t r o l}$ vs. CIN, p=0.000; control vs. SCC, $p=0.000$. ${ }^{\mathrm{b}}$ Control vs.CIN, $\mathrm{p}=0.000$; control vs. SCC, $\mathrm{p}=0.000$; CIN vs. SCC, $\mathrm{p}=0.002$. ${ }^{\mathrm{c}} \mathrm{Control}$ vs. CIN, $\mathrm{p}=0.000$; control vs. SCC, $\mathrm{p}=0.010 .{ }^{\mathrm{d}} \mathrm{C}$ ontrol vs. $\mathrm{CIN}, \mathrm{p}=0.000$; control vs. $\mathrm{SCC}, \mathrm{p}=0.000$.

expression levels of HLA-DR on PBMs from patients with malignancies of different origins, including lung cancer, colorectal cancer $(12,13)$ and glioblastoma (14), as well as head and neck cancer (15). Other authors, however, have failed to confirm these observations and found that PBMs from breast cancer patients did not differentially express HLA-DR when compared with those from healthy donors, but showed a higher transmigratory potency when interacting with the endothelial cells (16).

In the present study, PBMs from patients with CIN II-III and SCC were analyzed and an increase in HLA-DR expression was found in patients compared with the healthy controls. Additionally, the present study has demonstrated a significantly increased expression of HLA-DQ, another surface molecule belonging to the MHC class II complex, on PBMs from patients with CIN II-III and SCC. However, a markedly increased expression of HLA-DQ could be observed in patients with CIN II-III compared with SCC, but no significant differences existed between CIN II-III and SCC with regard to the expression of HLA-DR and HLA-DP. It has been shown that monocytes are capable of being heterogeneously activated by ligation to different MHC class II molecules, leading to a differential secretion of monokines, which may alter T-cell responses in vivo (17).

Thus, the upregulation of these molecules may contribute to an impaired antigen-presenting function of PBMs during the development of cervical cancer. However, other studies have reported that the low expression pattern of MHC class II may merely reflect an immunosuppressive environment induced by cytokines such as IL-10 or TGF- $\beta(18,19)$. However, this potential mechanism has to be further elucidated.

The classical HLA class I molecules are expressed on the surface of the majority of mammalian cells with only a few exceptions (20). Our results revealed that there were no significant changes in the expression of HLA class I on PBMs in patients with SCC and CIN II-III compared with the healthy control subjects.

As for the costimulatory surface molecules, we found a significantly increased expression of CD86 on PBMs from patients with SCC and CIN II-III compared with the healthy control subjects. By contrast, CD80 was only weakly expressed on PBMs, and revealed no significant differences among SCC, CIN II-III and the healthy controls. Both molecules of the B7 family, particularly CD86, play crucial roles in T-cell activation by APCs (21). These molecules were essential in demonstrating T-cell anergy in the absence of B7 signals (22). Thus, we suggest that the markedly increased expression of CD86 on PBMs from patients with SCC and CIN II-III found in our study indicates an increased costimulation and effector activation of T cells by PBMs, contributing to an increased antigen-specific immune response status in SCC and CIN II-III. Notably, it has been shown that low expression levels of CD86 on PBMs were significantly associated with unresponsiveness to vaccination against hepatitis B in chronic hemodialysis patients (23). This observation emphasizes the importance of our results, suggesting a possible impact for therapeutic vaccination strategies in cervical cancer patients.

T-cell activation causes the CD58-bound CD2 to be recognized and immobilized at sites of cell-cell contact, thereby strengthening T cell-APC adhesion (24). The expression levels of adhesion molecules by leukocytes were found to be important in their circulation and activation of leukocytes. Our results showed that the cell surface expression levels of CD58 were increased on PBMs from patients with SCC and CIN II-III compared with the healthy controls; however, there was no significant change between the expression levels of SCC and CIN II-III.

CD54, the intercellular adhesion molecule-1 (ICAM-1) is expressed in various immune cells, including lymphocytes or NK-cells, indicating that involvement in numerous immunological events including tumor cell-killer cell interactions. CD54 also enhances the major histocompatibility complex (MHC) peptide activation of $\mathrm{CD}^{+} \mathrm{T}$ cells without an organized immunological synapse and enhances antibody-mediated lysis of tumor cells through a lymphocyte function-associated antigen-1 (LFA-1)-dependent mechanism. Our results have shown that there were no significant differences in the expression levels of ICAM-1 among the three groups.

In conclusion, upregulated expression levels of HLA-DR, HLA-DQ, CD86/B7-2 and CD58 were associated with disease progression. These results indicate that increased expression 
levels of HLA-DR, HLA-DQ, CD86/B7-2 and CD58 on PBMs may be associated with the evolution of cervical cancer.

However, the role of these immunomodulators in regulating other costimulatory factors responsible for antigen presentation and lymphocyte activation should be investigated. The functional relevance of our findings remains to be elucidated.

\section{Acknowledgements}

This study was supported by funds from the Zhejiang Natural Science Foundation of China (Y2090355) and the Specialized Research Fund for the Doctoral Program of Higher Education (20090101120134).

\section{References}

1. Moore MA and Tajima K: Cervical cancer in the asian pacificepidemiology, screening and treatment. Asian Pac J Cancer Prev 5: 349-361, 2004.

2. Walboomers JM, Jacobs MV, Manos MM, et al: Human papillomavirus is a necessary cause of invasive cervical cancer worldwide. J Pathol 189: 12-19, 1999.

3. Chouaib S, Asselin-Paturel C, Mami-Chouaib F, Caignard A and Blay JY: The host-tumor immune conflict: from immunosuppression to resistance and destruction. Immunol Today 18 : 493-497, 1997

4. Zhou JH, Ye F, Chen HZ, Zhou CY, Lu WG and Xie X: Altered expression of cellular membrane molecules of HLA-DR, HLA-G and CD99 in cervical intraepithelial neoplasias and invasive squamous cell carcinoma. Life Sci 78: 2643-2649, 2006.

5. Huang AY, Golumbek P, Ahmadzadeh M, Jaffee E, Pardoll D and Levitsky H: Role of bone marrow-derived cells in presenting MHC class I-restricted tumor antigens. Science 264: 961-965, 1994.

6. Ardavin C, Martinez del Hoyo G, Martin P, et al: Origin and differentiation of dendritic cells. Trends Immunol 22: 691-700, 2001.

7. Fidler IJ and Kleinerman ES: Lymphokine-activated human blood monocytes destroy tumor cells but not normal cells under cocultivation conditions. J Clin Oncol 2: 937-943, 1984.

8. Kleinerman ES, Ceccorulli LM, Bonvini E, Zicht R and Gallin JI: Lysis of tumor cells by human blood monocytes by a mechanism independent of activation of the oxidative burst. Cancer Res 45: 2058-2064, 1985.

9. Ditschkowski M, Kreuzfelder E, Rebmann V, et al: HLA-DR expression and soluble HLA-DR levels in septic patients after trauma. Ann Surg 229: 246-254, 1999.

10. Gabrilovich DI, Corak J, Ciernik IF, Kavanaugh D and Carbone DP: Decreased antigen presentation by dendritic cells in patients with breast cancer. Clin Cancer Res 3: 483-490, 1997.
11. Pinzon-Charry A, Ho CS, Maxwell T, et al: Numerical and functional defects of blood dendritic cells in early- and late-stage breast cancer. Br J Cancer 97: 1251-1259, 2007.

12. Novellino PS, Trejo YG, Beviacqua M, Bordenave RH and Rumi LS: Cisplatin containing chemotherapy influences HLA-DR expression on monocytes from cancer patients. J Exp Clin Cancer Res 18: 481-484, 1999.

13. Novellino PS, Trejo YG, Beviacqua M, Bordenave RH and Rumi LS: Regulation of HLA-DR antigen in monocytes from colorectal cancer patients by in vitro treatment with human recombinant interferon-gamma. J Investig Allergol Clin Immunol 10: 90-93, 2000.

14. Woiciechowsky C, Asadullah K, Nestler D, et al: Diminished monocytic HLA-DR expression and ex vivo cytokine secretion capacity in patients with glioblastoma: effect of tumor extirpation. J Neuroimmunol 84: 164-171, 1998.

15. van Bokhorst-De van der Schuer MA, von Blomberg-van der Flier BM, Riezebos RK, et al: Differences in immune status between well-nourished and malnourished head and neck cancer patients. Clin Nutr 17: 107-111, 1998.

16. Gebhard B, Gnant M, Schutz G, et al: Different transendothelial migration behaviour pattern of blood monocytes derived from patients with benign and malignant diseases of the breast. Anticancer Res 20: 4599-4604, 2000.

17. Matsuoka T, Tabata H and Matsushita S: Monocytes are differentially activated through HLA-DR, -DQ, and -DP molecules via mitogen-activated protein kinases. J Immunol 166: 2202-2208, 2001.

18. Becker JC, Czerny C and Brocker EB: Maintenance of clonal anergy by endogenously produced IL-10. Int Immunol 6: 1605-1612, 1994.

19. Wahl SM, McCartney-Francis $\mathrm{N}$ and Mergenhagen SE: Inflammatory and immunomodulatory roles of TGF-beta. Immunol Today 10: 258-261, 1989.

20. Le Bouteiller P: HLA class I chromosomal region, genes, and products: facts and questions. Crit Rev Immunol 14: 89-129, 1994.

21. Van Gool SW, Vandenberghe P, de Boer M and Ceuppens JL: CD80, CD86 and CD40 provide accessory signals in a multiplestep T-cell activation model. Immunol Rev 153: 47-83, 1996.

22. Harding FA, McArthur JG, Gross JA, Raulet DH and Allison JP: CD28-mediated signalling co-stimulates murine T cells and prevents induction of anergy in T-cell clones. Nature 356: 607-609, 1992.

23. Girndt M, Sester M, Sester U, Kaul H and Kohler H: Defective expression of B7-2 (CD86) on monocytes of dialysis patients correlates to the uremia-associated immune defect. Kidney Int 59: 1382-1389, 2001

24. Zhu DM, Dustin ML, Cairo CW, Thatte HS and Golan DE: Mechanisms of cellular avidity regulation in CD2-CD58mediated T cell adhesion. ACS Chem Biol 1: 649-658, 2006. 